\title{
Dose Optimization in Pediatric Studies: Why It Is Important and How It Can Benefit Every Nuclear Medicine Department
}

\author{
John O. Prior ${ }^{1,2}$, Siroos Mirzaei ${ }^{2,3}$, Silvano Gnesin ${ }^{4}$, and Marie Nicod Lalonde ${ }^{1}$ \\ ${ }^{1}$ Department of Nuclear Medicine and Molecular Imaging, Lausanne University Hospital, Lausanne, Switzerland; ${ }^{2}$ Committee \\ on Accreditation of Nuclear Medicine Departments and Nuclear Medicine Training Centers, European Union of Medical \\ Specialists/European Board of Nuclear Medicine, Wien, Austria; ${ }^{3}$ Department of Nuclear Medicine and PET Center, Wiener \\ Gesundheitsverbund, Klinik Ottakring, Wien, Austria; and ${ }^{4}$ Applied Radiophysics Institute, Lausanne University Hospital, Lausanne, \\ Switzerland
}

See the associated article on page $\mathbf{5 7 0 .}$

I patient dose is suitable for the medical purpose and that unnecessary radiation is avoided. This principle of dose optimization is a general radiation protection principle applied in nuclear medicine and radiology and required by law in many countries (1). This principle is generally combined with the principle of level 2 justification, which ensures that the procedure is clinically indicated and traceable to national or international practice guidelines. Of course, exceptions are always possible when taking into account the singularities of a specific patient, such as a metallic implant or impaired renal function, impeding the realization of MRI and justifying the use of ionizing radiation in a young patient (level 3 justification). It is sensible to avoid unnecessary exposure to ionizing radiation in children, as they are more radiosensitive and have a longer life expectancy than adults.

There are 2 sets of international guidelines on recommended activities to inject in children: the North American Consensus Guidelines and the European Association of Nuclear Medicine pediatric dosage card. After obvious differences between these 2 guidelines were found by a comparison study (2), a new version of the pediatric dosage card was issued. Since then, it has been once more updated to its most current version (August 2016; https:// www.eanm.org/publications/dosage-card/) as an online calculator or smartphone application (PedDose App). As nuclear medicine instrumentation improves, such as with the introduction of PET silicon photomultiplier detectors, further modifications are expected.

In a study published in this issue of The Journal of Nuclear Medicine (3), Poli et al. undertook an International Atomic Energy Agency-coordinated research project to evaluate and optimize pediatric imaging in response to the existing divergence regarding the optimal activity for specific scintigraphic examinations. They

Received Aug. 24, 2020; revision accepted Sep. 9, 2020.

For correspondence or reprints contact: John O. Prior, Lausanne University Hospital, Nuclear Medicine and Molecular Imaging Department, Bugnon 46, $\mathrm{CH}-1011$ Lausanne, Switzerland.

E-mail: john.prior@chuv.ch

Published online Oct. 9, 2020.

COPYRIGHT (c) 2021 by the Society of Nuclear Medicine and Molecular Imaging. DOI: 10.2967/jnumed.120.254193 present their results for the procedure that has the highest impact in terms of dose optimization- ${ }^{99} \mathrm{~m}$ Tc-dimercaptosuccinic acid imaging. They applied a standard 5-step optimization method (3). Instead of the circle representation used in their study, we prefer to think of a staircase representation, with quality improvement occurring after completion of each cycle (Fig. 1).

The optimization methodology was very different for each of the 2 participating centers. At the first center (Department of Nuclear Medicine, Clinical Research Division of the Center of Isotopes, Havana, Cuba), the usual static acquisition was replaced by a dynamic one, allowing simulation of an image with a statistically lower count. This approach allowed the administered activity to be lowered by $30 \%-38 \%$, as compared with baseline, across different age categories. At the second center (Medscan Nuclear Medicine and PET/CT Center, Concepcion, Chile), a phantom approach led to the use of longer acquisition times than were usual for that center, allowing reductions of 50\%-70\% from baseline without loss of image quality. Consequently, Poli et al. concluded that their 5-step approach would allow centers to significantly decrease the radiation dose by $30 \%-70 \%$ without compromising image quality.

Instead of blindly applying guidelines that may not take into account the specific nuclear medicine instrumentation, $\gamma$-camera, and collimators at a particular center, centers can certainly use the proposed approach to help determine what level of reduction is possible without lowering image quality to an unacceptable degree. For instance, radiation doses might be reduced by longer acquisition times, which can be problematic in younger children who are more prone to move during the scan unless alternative strategies are used (immobilization device, sedation, or anesthesia, depending on the examination).

Projects that compare centers' administered activities in childreneven if pediatric nuclear medicine is not performed frequently-are worthwhile and an initial step toward continuous improvement. As stressed in the paper by Poli et al. (3), a multidisciplinary working group needs to be involved in this optimization process, with the nuclear medicine physician evaluating image quality and assessing the adequacy of the administered activity. On completion of each such optimization project, it might be worth embarking on another one, be it with the same radiopharmaceutical after another observation period or with a different radiopharmaceutical, depending on the frequency with which the clinic performs various studies. Two examples of projects that optimized radiation dose are the "Image Gently" initiative for children and "Image Wisely" initiative for adults.

Moreover, the proposed process for radiation dose optimization does not apply only to children but can benefit virtually any 
2. Collect injected activities \& image quality [BASELINE]

3. Compare activities with published guidelines

4. Define intervention

5. Implement \& evaluate [FOLLOW-UP]

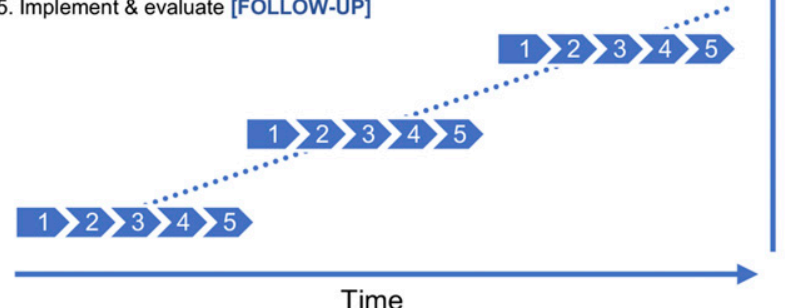

Time

FIGURE 1. Optimization methodology leading to improvement in quality after completion of each 5-step cycle. This methodology is in line with the well-known PDCA (Plan-Do-Check-Act) approach toward continuous quality improvement.

nuclear medicine department or practice. The proposed methodology of continuous quality improvement is consistent with the fundamental process of quality management in nuclear medicine. The International Atomic Energy Agency has introduced one such comprehensive audit program, freely available for self-administration by any nuclear medicine department, as the basis for ensuring the quality and safety of services for patients and staff, improving weaknesses, and reinforcing the strengths of nuclear medicine (4). Such audits can also be performed by external auditors and possibly declared mandatory by law in a specific country. The so-called clinical audits have been introduced as a tool to improve health protection against ionizing radiation by the Council of the European Union (1). Accordingly, member states of the European Atomic Energy Community are asked to implement national procedures to improve the quality of patient care and outcome. Procedures should be formally reviewed and compared with defined standards, and subsequent practice changes based on the results of this review should be implemented.

Accreditation of nuclear medicine departments has existed since 2000 across Europe and aims to deliver the highest possible level of quality (5). This accreditation takes place under the umbrella of the European Union of Medical Specialists and the European Board of Nuclear Medicine in close collaboration with the European Association of Nuclear Medicine (http://uems.eanm.org/). Accreditation compares what is done with what should be donelocal clinical standards are compared against national and international standards to ensure the correctness, consistency, and safety of day-to-day nuclear medicine practice. This process includes continuous improvement of radiation dose to patients, their families, and the nuclear medicine staff. Clinical audits and accreditation carry multiple further benefits, such as improving quality given the available resources; reducing hidden expenses, wastage, and duplication; revealing incorrect practices; and avoiding accidents or near-misses (5). In Europe, an accredited nuclear medicine department can also become an accredited nuclear medicine training center if it adopts the unified-training-requirement syllabus. Accreditation of training centers also ensures that high standards are maintained in nuclear medicine education.

To conclude, a simple optimization project such as the one presented by Poli et al. can identify suboptimal procedures in nuclear medicine diagnostics and, using an easily applicable methodology, bring about an immediate significant reduction in radiation dose without loss of image quality. Optimization has key implications for patient care and even greater implications for nuclear medicine by starting a cycle of continuous quality improvement that can lead to further changes in practice. This continuous improvement is what is required in a rapidly changing field such as nuclear medicine to deliver the highest-quality patient care.

\section{DISCLOSURE}

No potential conflict of interest relevant to this article was reported.

\section{REFERENCES}

1. Council Directive 2013/59/EURATOM of 5 December 2013 laying down basic safety standards for protection against the dangers arising from exposure to ionising radiation, and repealing Directives 89/618/Euratom, 90/641/Euratom, 96/29/ Euratom, 97/43/Euratom and 2003/122/Euratom. EUR-Lex.europa.eu website. https://eur-lex.europa.eu/eli/dir/2013/59/oj. Accessed December 3, 2020.

2. Grant FD, Gelfand MJ, Drubach LA, Treves ST, Fahey FH. Radiation doses for pediatric nuclear medicine studies: comparing the North American consensus guidelines and the pediatric dosage card of the European Association of Nuclear Medicine. Pediatr Radiol. 2015;45:706-713.

3. Poli GL, Coca M, Torres L, et al. Developing and implementing an imaging optimization study in pediatric nuclear medicine: experience and recommendations from an IAEA-coordinated research project. J Nucl Med. 2021;62:570-576.

4. Dondi M, Torres L, Marengo M, et al. Comprehensive auditing in nuclear medicine through the International Atomic Energy Agency Quality Management Audits in Nuclear Medicine (QUANUM) program. Part 1: the QUANUM program and methodology. Semin Nucl Med. 2017;47:680-686.

5. García-Burillo A, Hilson A, Mirzaei S. Why do we need accreditation of nuclear medicine departments? Eur J Nucl Med Mol Imaging. 2012;39:1643-1645. 